\title{
Imaging of the Paranasal Sinuses
}

While the preferred initial imaging modality of sinonasal disease has traditionally been computed tomography (CT), magnetic resonance imaging (MRI) has demonstrated several unique benefits for examination of the sinonasal cavities. Specifically, MRI, in most instances, can better distinguish between soft tissues when compared with CT. The distinction of soft tissues becomes most clinically relevant when evaluating for possible sinonasal tumors and when inspecting for extension of tumor or inflammation into the orbit or intracranial spaces. It is in these areas that MRI has found its major application in sinus imaging. This unit presents MRI techniques for imaging the sinuses with (see Basic Protocol 2) and without (see Basic Protocol 1) contrast enhancement. The parameters given here are derived from experience at $1.5 \mathrm{~T}$ and may need to be slightly altered depending on the field strength and the equipment manufacturer.

\section{IMAGING OF THE PARANASAL SINUSES BY MRI WITHOUT CONTRAST ENHANCEMENT}

Magnetic resonance imaging scans can be performed at a range of field strengths. Generally, the high signal-to-noise ratio obtained at high-field strengths allows one to scan either faster or with higher resolution when compared with lower field strengths. The sequences described within this unit are based on the authors' experience with a Siemens 1.5 T Symphony scanner, but are expected to be equally applicable to machines from other manufacturers.

Scanning a patient or volunteer is a team effort between technologists, nurses, and physicians. In most cases, the technologist assumes the lead role in acquiring the appropriate images.

The following set of sequences comprises the preferred method of imaging the sinuses. The usual sequences employed include transverse $T_{1}$-weighted spin echo and fat-saturated $T_{2}$-weighted fast or turbo spin-echo (FSE) images without contrast agent administration. Unenhanced MRI may be sufficient for diagnosis in uncomplicated inflammatory disease if there is no suspicion that the sinonasal pathology has violated the skull base. However, contrast enhancement is frequently necessary to assess whether a tumor or more extensive sinonasal inflammation has violated the skull base. In cases of suspected skull-base invasion, contrast-enhanced imaging (see Basic Protocol 2) is helpful due to its ability to better define the intracranial margins of a lesion or to detect perineural spread of a tumor. If a contrast agent is not used, coronal $T_{1}$-weighted spin echo and $T_{2}$-weighted fast spin-echo scans should also be obtained. The duration for the imaging process including all preparations will be $\sim 35$ min if no contrast agent is administered. All of these sequences are non-breathholding.

Table A7.6.1 lists the hardware necessary to perform the examination along with the appropriate parameters. The available gradient strength will depend on the scanner, and the echo times given below may have to be varied accordingly.

NOTE: Be sure that technologists and nurses have immediate access to any emergency equipment that may be relevant to a given study, or that may be needed for a particular patient, such as crash carts or oxygen.

\section{Set up patient and equipment}

1. Interview the patient to ensure that he or she has no contraindications such as cardiac pacemakers or other implants containing ferromagnetic materials. Also, be sure to 
Table A7.6.1 Equipment Parameters

\begin{tabular}{ll}
\hline Coil type & $\begin{array}{l}\text { Circularly polarized head coil (or } \\
\text { phased-array neck coil, if available) }\end{array}$ \\
Gradient coil strength & $30 \mathrm{mT} / \mathrm{m}$ (or whatever the system permits) \\
Cardiac gating & No \\
Peripheral gating & For safety only \\
Respiratory gating & No \\
Respirator & If required by patient \\
Oxygen & If required by patient \\
Motion cushions & Useful \\
Contrast agent & Used in Basic Protocol 2 \\
\hline
\end{tabular}

find out if the patient has any health conditions that may require the presence of the special emergency equipment during the scanning procedure or any other necessary precautions.

Generally, standard screening forms (APPENDIX 1) are used for all patients scanned in a magnetic resonance system.

The presence of any ferromagnetic metals may be a health hazard to the patient when he or she is inside the magnet, and will also affect the imaging. If in doubt as to the exact composition of the items, it is best to exclude patients with any metal implants; see Shellock and Crues (1998), Shellock and Kanal (1998a,b), Shellock and Shellock (1998a,b) and Shellock (2001) for a discussion of what implants may be scanned safely using magnetic resonance.

Patients may be accompanied into the magnet room by a friend or family member who can sit in the room during the scan and comfort the patient as needed. This companion must be screened as well to ensure the absence of loose metal objects on the body or clothing.

2. If the procedure is a research protocol, have the patient sign any necessary consent forms.

3. Have the patient remove all jewelry and change into a gown to eliminate any metal that may be found in clothing.

4. Have the patient wash off any mascara or other make up to avoid local tissue heating and image artifacts.

5. Inform the patient about what will occur during the procedure, what he or she will experience while in the magnet, as well as how to behave. Issues to discuss include the following:

a. If earplugs or headphones are used to protect the ears from loud sounds produced by the gradients, the patient will be asked to wear these, but will be able to communicate with you at any time during the imaging.

b. The patient will be given a safety squeeze bulb or similar equipment to request assistance at any time (demonstrate how this works).

c. For good results, the patient should not talk, and should avoid or minimize swallowing or other movements during each scan-i.e., as long as the banging sounds continue. Between scans, talking and swallowing are allowed in most cases, but should be avoided when comparative positional studies are being performed; the patient will be informed when this is the case.

Imaging of the Paranasal Sinuses 
6. Have the patient lie down on the table. Either before or right after the patient lies down, set up any triggering devices or other monitoring equipment that is to be used.

7. Center the patient in a head coil at the region where the key information is desired. Make sure that the head is constrained to prevent motion.

Generally, the patient's head is fixed so that the head is horizontal (not tilted) along the axis of the MRI table. A dedicated head coil is employed.

8. If needed, a pillow may be placed under the knees to make the patient more comfortable.

9. Use the centering light centered on the nasion of the patient and put him or her into the center of the magnet.

Once this step has been performed, so long as the patient does not move on the table, the table itself can be moved and then replaced in the same position as before without jeopardizing the positioning of one scan relative to another.

10. If the patient is unable to hold still, provide an appropriate sedative.

\section{Sequence 1: The pilot scan}

11. To validate the patient's position, run a scout scan to ensure correct localization of subsequent data set acquisitions using the imaging parameters given in Table A7.6.2.

This sequence usually consists of the three orthogonal planes to allow localization. The images are also often used later to set up total coverage for the volume of interest.

Sequence 2: Transverse $T_{2}$-weighted, fat-saturated turbo spin echo (TSE)

12. Display all the coronal, sagittal, and transverse scout images in split window mode on the scan monitor.

13. Change the imaging parameters to those listed in Table A7.6.3.

Table A7.6.2 Primary Clinical Imaging Parameters for Sequence 1 (Pilot Scan)

\begin{tabular}{ll}
\hline Patient position & $\begin{array}{l}\text { Supine } \\
\text { Gradient echo }\end{array}$ \\
Scan type & Transverse, coronal, and sagittal \\
Imaging plane (orientation) & Laser light centered on nasion \\
Central slice or volume center & $\leq 5 \mathrm{msec}$ \\
Echo time $\left(T_{\mathrm{E}}\right)$ & $\leq 20 \mathrm{msec}$ \\
Repeat time $\left(T_{\mathrm{R}}\right)$ & $40^{\circ}$ \\
Flip angle $(\mathrm{FA})$ & $280 \mathrm{~mm}, 280 \mathrm{~mm}$ \\
Fields of view $\left(\mathrm{FOV}, \mathrm{FOV}_{\mathrm{y}}\right)$ & $2.19 \mathrm{~mm}, 2.19 \mathrm{~mm}$ \\
Resolution $(\Delta x, \Delta y)$ & 128,128 \\
Number of data points collected $\left(N_{\mathrm{x}}, N_{\mathrm{y}}\right)$ & $10 \mathrm{~mm}$ \\
Slice thickness $(\Delta z)$ & 1 per orientation \\
Number of slices & Not applicable \\
Slice gap & 1 \\
Number of acquisitions $\left(N_{\text {acq }}\right)$ & No \\
Swap read and phase encoding & Anterior-posterior for the transverse \\
Read direction & images; Left-right for the coronal \\
& image; and cranial-caudal for the \\
& sagittal image \\
Scan time & 9 sec \\
\hline
\end{tabular}


Table A7.6.3 Primary Clinical Imaging Parameters for Sequence 2 ( $T_{2}$-Weighted Transverse Fat-Saturated TSE)

\begin{tabular}{ll}
\hline Patient position & Supine \\
Scan type & Turbo spin echo \\
Imaging plane (orientation) & Transverse \\
Central slice or volume center & Centered on nasion \\
Echo time $\left(T_{\mathrm{E}}\right)$ & $62 \mathrm{msec}$ \\
Echo train length $(\mathrm{ETL})$ & 7 \\
Repeat time $\left(T_{\mathrm{R}}\right)$ & $4000 \mathrm{msec}$ \\
Flip angle $(\mathrm{FA})$ & $150^{\circ} \mathrm{a}$ \\
Fields of view $\left(\mathrm{FOV}, \mathrm{F}_{\mathrm{x}}\right.$, FOV & $180 \mathrm{~mm}, 180 \mathrm{~mm}$ \\
Resolution $(\Delta x, \Delta y)$ & $0.70 \mathrm{~mm}, 0.70 \mathrm{~mm}$ \\
Number of data points collected $\left(N_{\mathrm{x}}, N_{\mathrm{y}}\right)$ & 256,256 \\
Slice thickness $(\Delta z)$ & $4 \mathrm{~mm}$ \\
Number of slices & 25 \\
Number of slabs & 1 \\
Slice gap & $0.8 \mathrm{~mm}$ \\
Number of acquisitions $\left(N_{\mathrm{acq}}\right)$ & 2 \\
Swap read and phase encoding & No \\
Read direction & Right-left \\
Fat suppression & Yes \\
Scan time & $302 \mathrm{sec}$ \\
\hline${ }^{a}$ The system displays the flip angle of the refocusing pulse. The flip angle of the first pulse of this sequence \\
is $90^{\circ}$.
\end{tabular}

14. Position the slices from the scout images to cover the whole sinonasal region, from the tip of the nose to the posterior part of the sphenoid sinus in the transverse plane, from the superior margin of the frontal sinus to the palate in the sagittal plane, and covering both lateral margins of the maxillary sinuses. Run sequence 2 according to Table A7.6.3.

An example is shown in Fig. A7.6.1.

\section{Sequence 3: Transverse $T_{1}$-weighted spin echo (SE)}

15. Copy the imaging parameters such as number of slices, field of view, thickness of slices, and center of slices from the performed transverse $T_{2}$-weighted fat-saturated sequence (sequence 2) and change the imaging parameters to those listed in Table A7.6.4. Run sequence 3 according to Table A7.6.4.

An example is shown in Fig. A7.6.2.

\section{Sequence 4: Coronal $T_{1}$-weighted spin echo}

16. Display the transverse images (use images from sequence 2 or 3 ) on the scan monitor.

17. Change the imaging parameters to those listed in Table A7.6.5. Position slices to center of the $T_{1}$ - or $T_{2}$-weighted spin echo image ensuring that the sinuses are fully covered. Run sequence 4 according to Table A7.6.5.

An example is shown in Fig. A7.6.3.

\section{Sequence 5: Coronal $T_{2}$-weighted, fat-saturated turbo spin echo}

18. Copy the imaging parameters such as number of slices, field of view, thickness of slices and center of slices from the performed coronal $T_{1}$-weighted sequence (sequence 4) and change the imaging parameters to those listed in Table A7.6.6. Run sequence 5 according to Table A7.6.6. 


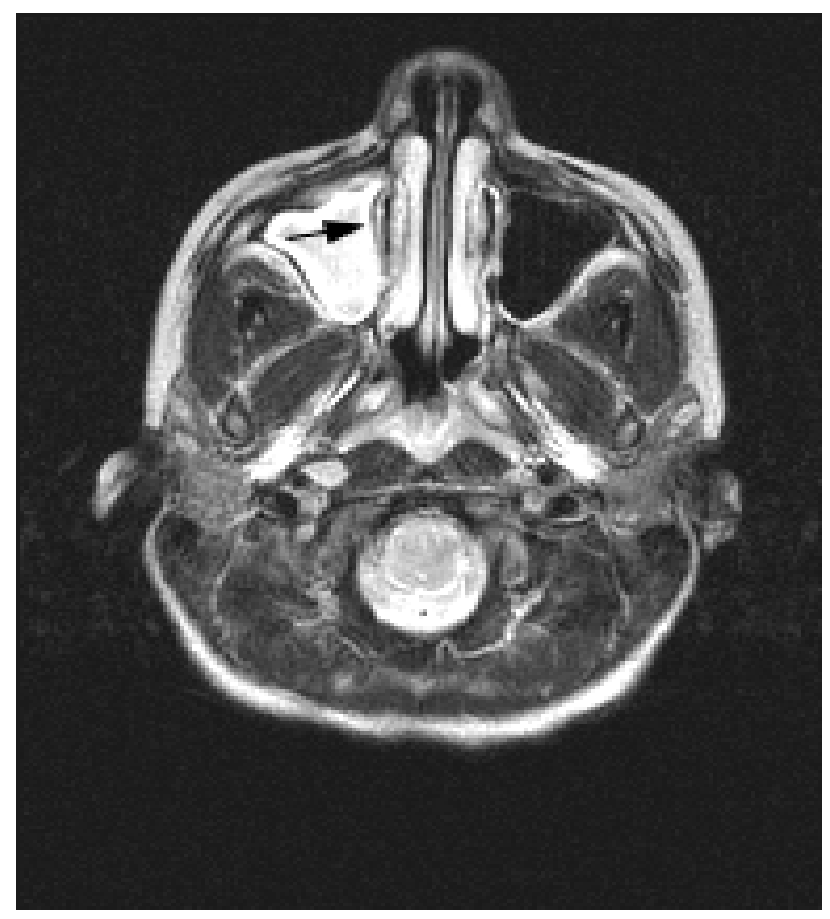

Figure A7.6.1 This $T_{2}$-weighted TSE image shows an opacification (arrow) of the right maxillary sinus.

Table A7.6.4 Primary Clinical Imaging Parameters for Sequence 3 (Transverse $T_{1}$-Weighted Spin Echo)

$\begin{array}{ll}\text { Patient position } & \text { Supine } \\ \text { Scan type } & \text { Spin echo } \\ \text { Imaging plane (orientation) } & \text { Transverse } \\ \text { Central slice or volume center } & \text { Centered on nasion } \\ \text { Echo time }\left(T_{\mathrm{E}}\right) & 10 \mathrm{msec} \\ \text { Repeat time }\left(T_{\mathrm{R}}\right) & 532 \mathrm{msec} \\ \text { Flip angle }(\mathrm{FA}) & 90^{\circ} \\ \text { Fields of view }\left(\mathrm{FOV}_{\mathrm{x}}, \mathrm{FOV}_{\mathrm{y}}\right) & 180 \mathrm{~mm}, 180 \mathrm{~mm} \\ \text { Resolution }(\Delta x, \Delta y) & 0.70 \mathrm{~mm}, 0.70 \mathrm{~mm} \\ \text { Number of data points collected }\left(N_{\mathrm{x}}, N_{\mathrm{y}}\right) & 256,256^{a} \\ \text { Slice thickness }(\Delta z) & 4 \mathrm{~mm} \\ \text { Number of slices } & 25 \\ \text { Number of slabs } & 1 \\ \text { Slice gap } & 0.8 \mathrm{~mm} \\ \text { Number of acquisitions }\left(N_{\mathrm{acq}}\right) & 2 \\ \text { Swap read and phase encoding } & \text { No } \\ \text { Read direction } & \text { Right-left } \\ \text { Scan time } & 242 \mathrm{sec}\end{array}$

${ }^{a}$ The true number of collected phase encoding lines is 224 , a reduction due to a partial Fourier factor of $7 / 8$. 


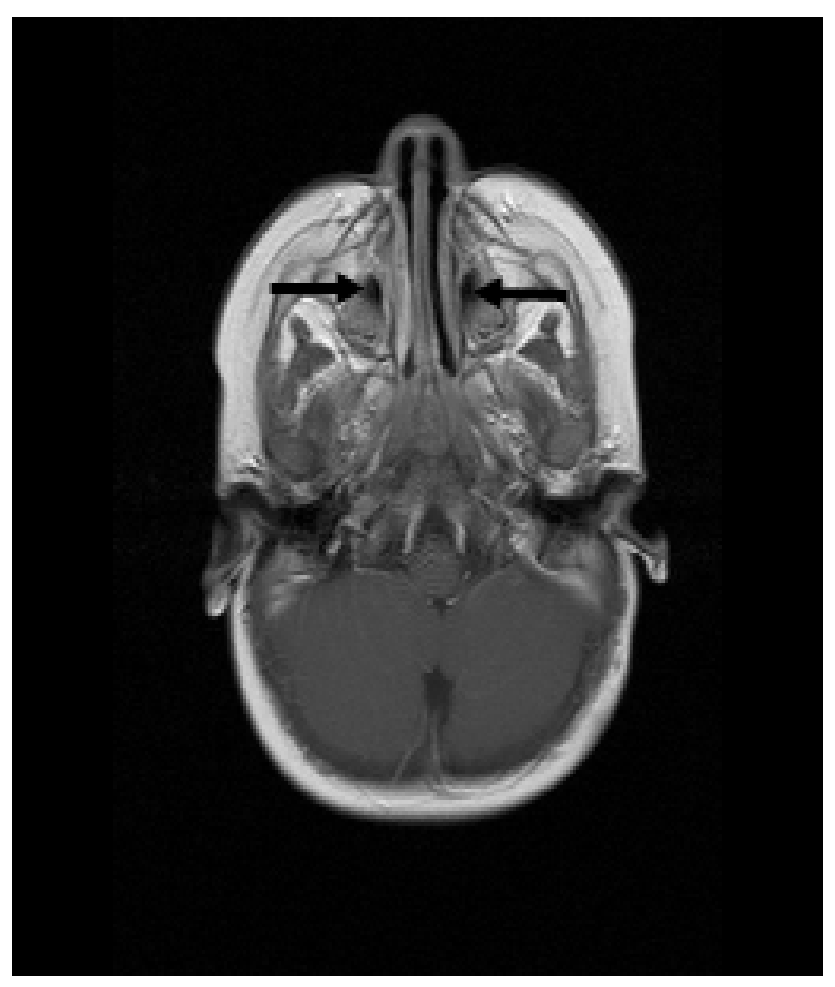

Figure A7.6.2 This $T_{1}$-weighted SE image shows the maxillary sinus of an 8-month-old male.

Table A7.6.5 Primary Clinical Imaging Parameters for Sequence 4 (Coronal $T_{1}$-Weighted Spin Echo)

\begin{tabular}{ll}
\hline Patient position & Supine \\
Scan type & Spin echo \\
Imaging plane (orientation) & Coronal \\
Central slice or volume center & Centered on nasion \\
Echo time $\left(T_{\mathrm{E}}\right)$ & $10 \mathrm{msec}$ \\
Repeat time $\left(T_{\mathrm{R}}\right)$ & $532 \mathrm{msec}$ \\
Flip angle (FA) & $90^{\circ}$ \\
Fields of view $\left(\mathrm{FOV}_{\mathrm{x}}, \mathrm{FOV}_{\mathrm{y}}\right)$ & $180 \mathrm{~mm}, 180 \mathrm{~mm}$ \\
Resolution $(\Delta x, \Delta y)$ & $0.70 \mathrm{~mm}, 0.70 \mathrm{~mm}$ \\
Number of data points collected $\left(N_{\mathrm{x}}, N_{\mathrm{y}}\right)$ & $256,256^{a}$ \\
Slice thickness $(\Delta z)$ & $4 \mathrm{~mm}$ \\
Number of slices & 19 \\
Number of slabs & 1 \\
Slice gap & $0.8 \mathrm{~mm}$ \\
Number of acquisitions $\left(N_{\mathrm{acq}}\right)$ & 2 \\
Swap read and phase encoding & No \\
Read direction & Right-left \\
Scan time & $242 \mathrm{sec}$ \\
\hline
\end{tabular}

$a_{\text {The true number of collected phase encoding lines is } 224 \text {, a reduction due to a partial Fourier factor }}$ of $7 / 8$. 


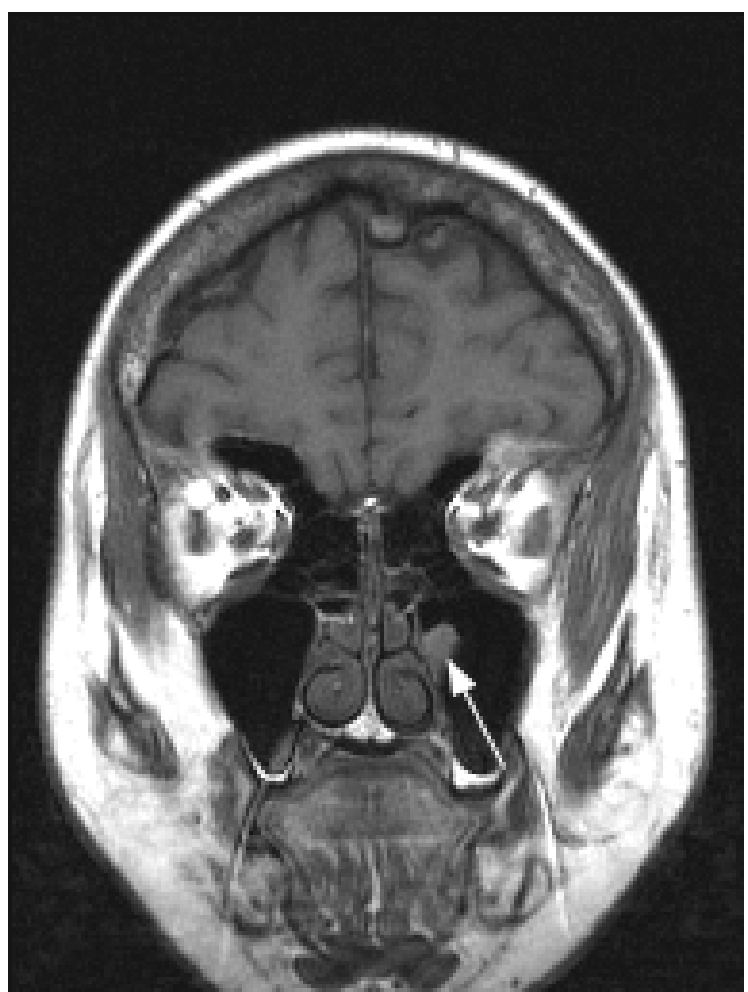

Figure A7.6.3 This $T_{1}$-weighted SE image shows a small retention cyst or polyp (arrow) within the left maxillary sinus.

Table A7.6.6 Primary Clinical Imaging Parameters for Sequence 5 (Coronal $T_{2}$-Weighted Fat-Saturated TSE)

\begin{tabular}{ll}
\hline Patient position & Supine \\
Scan type & Turbo spin echo \\
Imaging plane (orientation) & Coronal \\
Central slice or volume center & Centered on nasion \\
Echo time $\left(T_{\mathrm{E}}\right)$ & $62 \mathrm{msec}$ \\
Echo train length $(\mathrm{ETL})$ & 7 \\
Repeat time $\left(T_{\mathrm{R}}\right)$ & $4000 \mathrm{msec}$ \\
Flip angle $(\mathrm{FA})$ & $150^{\circ} \mathrm{a}$ \\
Fields of view $\left(\mathrm{FOV}, \mathrm{V}_{\mathrm{x}}, \mathrm{FO}_{\mathrm{y}}\right)$ & $180 \mathrm{~mm}, 180 \mathrm{~mm}$ \\
Resolution $(\Delta x, \Delta y)$ & $0.70 \mathrm{~mm}, 0.70 \mathrm{~mm}$ \\
Number of data points collected $\left(N_{\mathrm{x}}, N_{\mathrm{y}}\right)$ & 256,256 \\
Slice thickness $(\Delta z)$ & $4 \mathrm{~mm}$ \\
Number of slices & 19 \\
Number of slabs & 1 \\
Slice gap & $0.8 \mathrm{~mm}$ \\
Number of acquisitions $\left(N_{\mathrm{acq}}\right)$ & 2 \\
Swap read and phase encoding & No \\
Read direction & Right-left \\
Fat suppression & Yes \\
Scan time & $302 \mathrm{sec}$ \\
\hline$a_{\text {The system displays the flip angle of the refocusing pulse. The flip angle of the first pulse of this sequence }}$ \\
is $90^{\circ}$.
\end{tabular}


Table A7.6.7 Primary Clinical Imaging Parameters for Sequence 6 (Transverse

$T_{1}$-Weighted Fat-Saturated Spin Echo)

Patient position

Scan type

Imaging plane (orientation)

Central slice or volume center

Echo time $\left(T_{\mathrm{E}}\right)$

Repeat time $\left(T_{\mathrm{R}}\right)$

Flip angle (FA)

Fields of view $\left(\mathrm{FOV}_{\mathrm{x}}, \mathrm{FOV}_{\mathrm{y}}\right)$

Resolution $(\Delta x, \Delta y)$

Number of data points collected $\left(N_{\mathrm{x}}, N_{\mathrm{y}}\right)$

Slice thickness $(\Delta z)$

Number of slices

Number of slabs

Slice gap

Number of excitations (NEX)

Number of acquisitions $\left(N_{\text {acq }}\right)$

Swap read and phase encoding

Read direction

Fat suppression

Scan time
Supine

Spin echo

Transverse

Centered on nasion

$10 \mathrm{msec}$

$500 \mathrm{msec}$

$90^{\circ}$

$180 \mathrm{~mm}, 180 \mathrm{~mm}$

$0.70 \mathrm{~mm}, 0.70 \mathrm{~mm}$

$256,256^{a}$

$4 \mathrm{~mm}$

25 (total)

2

$0.8 \mathrm{~mm}$

$2^{b}$

2

No

Right-left

Yes

$390 \mathrm{sec}$
BASIC PROTOCOL 2

Imaging of the Paranasal Sinuses

A7.6.8

${ }^{a}$ The true number of collected phase encoding lines is 192, a reduction due to a partial Fourier factor of $3 / 4$.

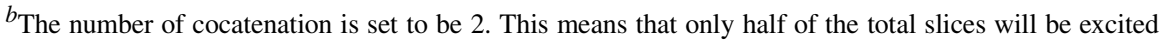
during a given repeat time.

\section{IMAGING OF THE PARANASAL SINUSES BY MRI WITH CONTRAST ENHANCEMENT}

In cases of suspected skull base, intracranial, intraorbital, or perineural extension of sinonasal pathology, the administration of a contrast agent is advised. In addition, some have suggested a role for post-contrast images if a solid mass cannot be distinguished from a cystic lesion on the non-contrast-enhanced examination, as characteristic enhancement of a cyst wall can often be appreciated. If a contrast agent is employed, transverse and coronal $T_{1}$-weighted, fat-suppressed, contrast-enhanced sequences should be obtained. If a contrast agent is administered, the duration of the imaging process including all preparations will be $\sim 50 \mathrm{~min}$. All these sequences are non-breathholding.

\section{Materials}

Normal saline $(0.9 \% \mathrm{NaCl})$, sterile

Gadolinium-based MR contrast agent (e.g., Magnevist, Omniscan, or Prohance)

1. Perform Basic Protocol 1, steps 1 to 6.

2. Establish an intravenous line from which the contrast agent can be injected, and attach this line securely to the patient so that movement into or out of the magnet will not pull at the patient's arm.

It is preferable to insert the line prior to imaging and to leave the patient in the magnet, so that there is no intervening motion between the scans run before contrast agent injection and those run after injection.

3. Perform Basic Protocol 1, steps 7 to 10 .

4. Perform Basic Protocol 1, sequences 1, 2, and 3.

5. Administer the contrast agent. A dose of $0.2 \mathrm{mmol} / \mathrm{kg}$ gadolinium is usually given at a rate of $1 \mathrm{ml} / \mathrm{sec}$. 


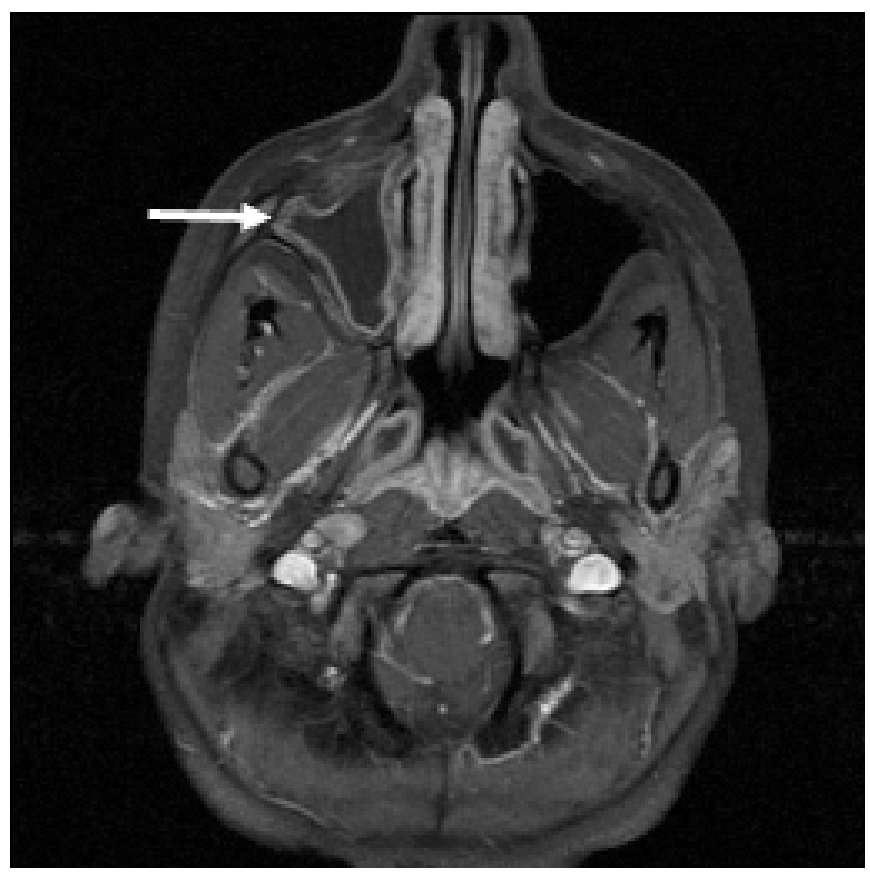

Figure A7.6.4 This $T_{1}$-weighted SE image with fat saturation shows contrast enhancement of the mucous membranes (arrow) of the opacified right maxillary sinus.

Table A7.6.8 Primary Clinical Imaging Parameters for Sequence 7 (Coronal $T_{1}$-Weighted Fat-Saturated Spin Echo)

\begin{tabular}{ll}
\hline Patient position & Supine \\
Scan type & Spin echo \\
Imaging plane (orientation) & Coronal \\
Central slice or volume center & Centered on nasion \\
Echo time $\left(T_{\mathrm{E}}\right)$ & $10 \mathrm{msec}$ \\
Repeat time $\left(T_{\mathrm{R}}\right)$ & $723 \mathrm{msec}$ \\
Flip angle $(\mathrm{FA})$ & $90^{\circ}$ \\
Fields of view $\left(\mathrm{FOV}, \mathrm{FOV}_{\mathrm{y}}\right)$ & $180 \mathrm{~mm}, 180 \mathrm{~mm}$ \\
Resolution $(\Delta x, \Delta y)$ & $0.70 \mathrm{~mm}, 0.70 \mathrm{~mm}$ \\
Number of data points collected $\left(N_{\mathrm{x}}, N_{\mathrm{y}}\right)$ & $256,256^{a}$ \\
Slice thickness $(\Delta z)$ & $4 \mathrm{~mm}$ \\
Number of slices & 19 \\
Number of slabs & 1 \\
Slice gap & $0.8 \mathrm{~mm}$ \\
Number of acquisitions $\left(N_{\text {acq }}\right)$ & 2 \\
Swap read and phase encoding & No \\
Read direction & Right-left \\
Fat suppression & Yes \\
Scan time & $281 \mathrm{sec}$ \\
\hline$a_{\text {The true number of collected phase encoding lines }}$ is 192, a reduction due to a partial Fourier factor \\
of 3/4.
\end{tabular}




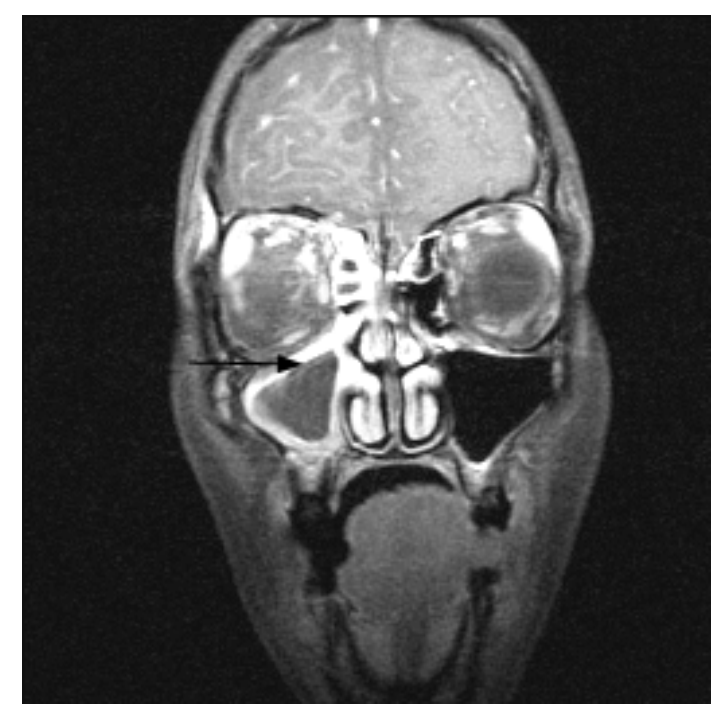

Figure A7.6.5 This $T_{1}$-weighted SE image with fat saturation shows contrast enhancement of the mucous membranes (arrow) of the opacified right maxillary sinus, same patient as in Figure A7.6.4.

6. Perform the following sequences 3 min post-injection.

\section{Sequence 6: Transverse $T_{1}$-weighted, fat-saturated spin echo}

7. Copy the imaging parameters such as number of slices, field of view, thickness of slices, and center of slices from the performed transverse $T_{1}$-weighted sequence (sequence 3) and change the imaging parameters to those listed in Table A7.6.7. Run sequence 6 according to Table A7.6.7.

An example is shown in Figure A7.6.4.

\section{Sequence 7: Coronal $T_{1}$-weighted, fat-saturated spin echo}

8. Copy the imaging parameters such as number of slices, field of view, thickness of slices, and center of slices from the performed coronal $T_{1}$-weighted sequence (sequence 4) and change the imaging parameters to those listed in Table A7.6.8. Run sequence 7 according to Table A7.6.8.

An example is shown in Fig. A7.6.5.

\section{COMMENTARY}

\section{Background Information}

The examination of the anatomy and pathology of paranasal sinuses, as seen by MRI as well as CT, shows a wide variety of findings in a range of $15 \%$ to $40 \%$ of all adults that undergo imaging (Conner et al., 1989). Some of the findings can be correlated to acute upper respiratory infection and allergic episodes with a typical seasonal pattern (Moore et al., 1986). But most of the abnormalities in the paranasal sinuses were unrelated to the patients' presenting problems (Moser et al., 1991) and are, therefore, often reported as incidental findings on scans performed for indications other than the evaluation of a paranasal sinus pathology. Thus, many people with inflammatory changes in their sinuses demonstrated on MRI do not have symptoms classically attributed to sinusitis (Cooke and Hadley, 1991).

The most common inflammatory abnormalities noted on imaging studies include opacified sinus cavities, intrasinus air-fluid levels and polyps, mucoperiosteal thickening, isolated increased signal of the mucosal lining, mucus retention cysts, and fungal concretions (Zinreich, 1990). Abnormalities were most commonly seen in the ethmoid sinuses followed by the maxillary cavities, and, to a lesser extent, in the sphenoid and frontal sinuses (Wani et al., 2001).

Findings of high significance on paranasal sinus studies are mastoiditis as a known cause
Imaging of the

A7.6.10 
of lateral venous sinus thrombosis (Fink and McAuley, 2002), and sphenoid mucoceles. Although benign, sphenoid mucoceles may involve the orbit and cause acute restrictive ophthalmoplegia, proptosis, and reduced visual acuity (Conner et al., 1989).

Malignant pathologies involving the paranasal sinuses are rare, but nasopharyngeal carcinoma may spread to the paranasal sinuses. Maxillary sinus infiltration by this tumor is most common but contrast-enhanced MRI allows differentiating tumor from inflammatory changes in all sinuses (Chong et al., 1998). MR imaging is also indicated for the evaluation of primary tumors of the sinuses and nasal cavity, including squamous cell carcinoma, esthesioneuroblastoma, lymphoma, and inverted papilloma. In the presence of an aggressive tumor, particular attention should be directed toward dural thickening or invasion, periorbital invasion, or perineural extension. In addition, the pterygopalatine fossa should be examined for evidence of posterior direct or perineural extension in the presence of maxillary sinus or hard palate involvement by tumor.

Magnetic resonance imaging has proven to be a useful tool for imaging the paranasal sinuses and nasal cavities. Of particular interest in this region is the ability to distinguish lesions that are highly cellular with little free water, demonstrating intermediate signal on $T_{2}$ weighted images, most likely representing tumor, from those lesions that have significant amounts of serous and mucinous secretions, very high in signal on $T_{2}$-weighted images, that more likely represent an inflammatory or postobstructive process containing predominantly free water (Hasso and Lambert, 1994).

Furthermore, the multiplanar capabilities of MRI allow identifying tumor margins demonstrating the extent of the disease and specifically the relationship to key landmarks critical for further surgical treatment (Weissman et al., 1990).

CT remains the examination of choice for evaluation of the thin cortical bones of the osteomeatal complex, and for visualization of osteolytic regions with high sensitivity and spatial resolution (Weissman et al., 1990). Another indication for CT is for evaluation of osteomas involving the paranasal sinuses (Beahm et al., 1990).

\section{Critical Parameters and Troubleshooting}

The high magnetic field strength of $1.5 \mathrm{~T}$ magnets and $T_{2}$-weighted spin echo techniques reveal a remarkably intense signal from abnor- mal tissue in the paranasal sinuses. Inflammatory disease in the maxillary, sphenoid, ethmoid, and frontal sinuses is detected and demonstrated with greater clarity than any other available technique. The additional administration of gadolinium as a contrast agent allows detection and characterization of malignant processes and their margins, along with detection of extension and infiltration when fat-saturated $T_{1}$-weighted spin echo techniques are applied (Moore et al., 1986).

The majority of the pathological processes can be adequately imaged by transverse $T_{1}$ - and $T_{2}$-weighted pulse sequences. When more tissue specific information is required, additional coronal and sagittal planes are helpful for tumors that infiltrate the skull base or are surrounded by air-filled sinuses (Beahm et al., 1990).

High spatial resolution is desired so that small areas of signal variation mostly due to edema are not missed. It is also important to center the slice-selecting planes in the transverse, coronal, and sagittal orientation to perform a comparison with the opposite side in order to identify any area of abnormality and differentiate between pathology and anatomical variation.

\section{Anticipated Results}

A variety of findings within the paranasal sinuses can be identified utilizing magnetic resonance imaging and computed tomography.

The primary role of CT is in visualizing the thin cortical bones of the osteomeatal complex in order to precisely identify bony abnormalities with high sensitivity and spatial resolution.

MRI is considered by many the imaging modality of choice for evaluating any soft-tissue lesion of the paranasal sinuses. These lesions may range from rather less significant importance for the patient with no further treatment such as mild sinus inflammation, polyps, mucus retention cysts, mucoperiosteal thickening, or simple inflammatory increased signal of the epithelial lining, to less common lesions such as fungal concretions, sphenoid mucoceles, and infections such as mastoiditis, which may result in further even life threatening complications.

Although a specific diagnosis can be suggested in most cases by other means of visualization, only MRI has the potential to demonstrate the extent of the disease with high precision.

The visualization of malignancies such as nasopharyngeal carcinoma, sinus carcinoma,
Head and Neck A7.6.11

Supplement 7 
lymphoma, esthesioneuroblastoma, or nasal cavity tumors can be accomplished with a maximum degree of spatial resolution by MRI and thereby help to identify routes of tumor spread and key surgical landmarks for further therapy.

\section{Literature Cited}

Beahm, E., Teresi, L., Lufkin, R., and Hanafee, W. 1990. MR of the paranasal sinuses. Surg. Radiol. Anat. 12:203-208.

Chong, V.F., Fan, Y.F., and Khoo, J.B. 1998. Computed tomographic and magnetic resonance imaging findings in paranasal sinus involvement in nasopharyngeal carcinoma. Ann. Acad. Med. Singapore 27:800-804.

Conner, B.L., Roach, E.S., Laster, W., and Georgitis, J.W. 1989. Magnetic resonance imaging of the paranasal sinuses: Frequency and type of abnormalities. Ann. Allergy 62:457-460.

Cooke, L.D. and Hadley, D.M. 1991. MRI of the paranasal sinuses: Incidental abnormalities and their relationship to symptoms. J. Laryngol. Otol. 105:278-281.

Fink, J.N. and McAuley, D.L. 2002. Mastoid air sinus abnormalities associated with lateral venous sinus thrombosis: Cause or consequence? Stroke 33:290-292.

Hasso, A.N. and Lambert, D. 1994. Magnetic resonance imaging of the paranasal sinuses and nasal cavities. Top Magn. Reson. Imaging 6:209-223.

Moore, J., Potchen, M., Waldenmaier, N., Sierra, A. and Potchen, E.J. 1986. High-field magnetic resonance imaging of paranasal sinus inflammatory disease. Laryngoscope 96:267-271.

Moser, F.G., Panush, D., Rubin, J.S., Honigsberg, R.M., Sprayregen, S., and Eisig, S.B. 1991. In- cidental paranasal sinus abnormalities on MRI of the brain. Clin. Radiol. 43:252-254.

Shellock, F.G. 2001. Pocket Guide to MR Procedures and Metallic Objects. Lippincott-Raven, Philadelphia.

Shellock, F.G. and Crues, J.V. 1998. Aneurysm clips: Assessment of magnetic field interaction associated with a 0.2-T extremity MR system. Radiology 208:407-409.

Shellock, F.G. and Kanal, E. 1998a. Aneurysm clips: Evaluation of MR imaging artifacts at $1.5 \mathrm{~T}$. Radiology 209:563-566.

Shellock, F.G. and Kanal, E. 1998b. Yasargil aneurysm clips: Evaluation of interactions with a 1.5-T MR system. Radiology 207:587-591.

Shellock, F.G. and Shellock, V.J. 1998a. Cranial bone flap fixation clamps: Compatibility at MR imaging. Radiology 207:822-825.

Shellock, F.G. and Shellock, V.J. 1998b. Spetzler titanium aneurysm clips: Compatibility at MR imaging. Radiology 206:838-841.

Wani, M.K., Ruckenstein, M.J., and Parikh, S. 2001. Magnetic resonance imaging of the paranasal sinuses: Incidental abnormalities and their relationship to patient symptoms. J. Otolaryngol 30:257-262.

Weissman, J.L., Tabor, E.K., and Curtin, H.D. 1990. Magnetic resonance imaging of the paranasal sinuses. Top. Magn. Reson. Imaging 2:27-38.

Zinreich, S.J. 1990. Paranasal sinus imaging. Otolaryngol Head Neck Surg. 103:863-868.

Contributed by Daniel T. Boll, Michael Coffey, and Jonathan S. Lewin

University Hospitals of Cleveland/ Case Western Reserve University Cleveland, Ohio 